\title{
Multitrace operators and the generalized AdS/CFT prescription
}

\author{
Pablo Minces* \\ Instituto de Física Teórica, Universidade Estadual Paulista, Rua Pamplona 145, 01405-900, São Paulo, SP, Brazil
}

(Received 2 December 2002; published 29 July 2003)

\begin{abstract}
We show that multitrace interactions can be consistently incorporated into an extended AdS conformal field theory (CFT) prescription involving the inclusion of generalized boundary conditions and a modified Legendre transform prescription. We find new and consistent results by considering a self-contained formulation which relates the quantization of the bulk theory to the AdS/CFT correspondence and the perturbation at the boundary by double-trace interactions. We show that there exist particular double-trace perturbations for which irregular modes are allowed to propagate as well as the regular ones. We perform a detailed analysis of many different possible situations, for both minimally and nonminimally coupled cases. In all situations, we make use of a new constraint which is found by requiring consistency. In the particular nonminimally coupled case, the natural extension of the Gibbons-Hawking surface term is generated.
\end{abstract}

DOI: $10.1103 /$ PhysRevD.68.024027

\section{INTRODUCTION}

In recent papers $[1,2]$, interest in the role of multitrace interactions in the AdS conformal field theory (CFT) correspondence [3] has been revived by introducing the proposal that to deform the boundary CFT by double-trace operators gives rise to a new perturbation expansion for string theory which is based on a nonlocal worldsheet.

In the AdS/CFT picture, multitrace operators in the boundary CFT are understood as multiparticle states in AdS (see, for example, [4,5]), and this raises the question of how to place a boundary condition on a multiparticle state and perform explicit calculations in the context of the AdS/CFT correspondence.

A solution to this problem came with the proposal in $[6,7]$ that multitrace operators can be incorporated by generalizing the usual Dirichlet prescription which is considered in the case of single-trace operators. Such prescription reads $[8,9]$

$$
\exp \left(-I_{\mathrm{AdS}}\left[\phi_{0}\right]\right) \equiv\left\langle\exp \left(\int d^{d} x O(\vec{x}) \phi_{0}(\vec{x})\right)\right\rangle,
$$

where $d+1$ is the dimension of the AdS bulk, and $\phi_{0}$ is the boundary value of the bulk field $\phi$ which couples to the boundary CFT operator $O$.

The perturbation of the boundary CFT by multitrace operators can be written schematically as

$$
I_{Q F T}[O]=I_{C F T}[O]+\int d^{d} x W[O],
$$

where $W[O]$ is an arbitrary function of $O$. In the particular case of a double-trace perturbation of the form

$$
W[O]=\frac{\beta}{2} O^{2},
$$

where $O$ has conformal dimension $\Delta=d / 2$, it has been shown in [6] that a generalized boundary condition gives rise to the correct renormalization formula for the coupling $\beta$.

*Email address: pablo@fma.if.usp.br
PACS number(s): 04.62.+v

The purpose of this paper is to introduce a further development of the proposal in $[6,7]$ by considering a generalized AdS/CFT prescription in which multitrace interactions can be consistently incorporated. More specifically, we aim to show that the recent proposal in [10] for a generalized AdS/ CFT prescription which makes use of generalized boundary conditions provides a natural frame for consistently describing multitrace operators in the AdS/CFT correspondence. For the sake of simplicity, we will concentrate on the particular case of double-trace interactions, for which we will relate the coupling coefficient to specific boundary conditions on the scalar field, or equivalently, to the addition of specific boundary terms to the usual bulk action. We will also interpret our results in terms of the energy of the theory in the bulk and the constraints which arise when performing its quantization. In addition, we will extend the formulation to the nonminimally coupled case.

In considering the quantization of the scalar field theory on AdS, it has been shown in [11-13] that there exist particular constraints on the mass and the coupling coefficient to the metric for which two different asymptotic behaviors of the field cause the energy to be conserved, positive, and finite. Such asymptotic behaviors are of the form

$$
\phi^{R} \sim \epsilon^{\Delta_{+}}, \quad \phi^{I} \sim \epsilon^{\Delta_{-}},
$$

where $\phi^{R}$ and $\phi^{I}$ stand for "regular" and "irregular" modes, $\epsilon$ is a measure of the distance to the boundary which is considered to be small, and

$$
\Delta_{ \pm}=\frac{d}{2} \pm \nu
$$

where

$$
\nu=\sqrt{\frac{d^{2}}{4}+M^{2}} .
$$

Here $M$ is the effective mass of the scalar field, and in the nonminimally coupled case it is given by

$$
M^{2}=m^{2}+\varrho R,
$$


where $R$ is the Ricci scalar of the metric (which is a negative constant) and $\varrho$ is the coupling coefficient. In the minimally coupled case $\varrho=0$, the above equation reduces to $M=m$.

Since there exist two possible quantizations of the theory on the bulk, we expect that there must exist two different boundary CFT's. However, it has been pointed out in [14] that the usual Dirichlet prescription Eq. (1) can only account for one of them, namely the one with conformal dimension $\Delta_{+}$, which corresponds to regular modes propagating in the bulk. In order to also account for the missing conformal dimension $\Delta_{-}$, the proposal in [14] is that its generating functional is the Legendre transform of the one which gives rise to the conformal dimension $\Delta_{+}$. It was explicitly shown in [14] that in fact this prescription gives rise to the missing conformal dimension $\Delta_{-}$.

Later, it was shown in [10] that there were still some problems which needed to be considered. One of them is the fact that the usual AdS/CFT prescription is unable to reproduce the constraints in $\varrho$ and $\nu$ for which the energy in [11-13] is conserved, positive, and finite for irregular modes propagating in the bulk. The second problem is that the usual Legendre transform prescription leaves a coefficient to be fixed "by hand." And the last problem regarding the usual Legendre transform is that it does not work for some particular values of $\nu$.

Then, the proposal in [10] was to consider a generalized formalism which involves modifications both in the bulk quantization and in the AdS/CFT prescription. From the bulk point of view, it was suggested in [10] that the natural energy to be considered in the AdS/CFT correspondence context is the "canonical" energy, which is constructed out of the Noether current corresponding to time translations, rather than the "metrical" one, which is constructed through the stressenergy tensor, as in [11-13]. The reason for considering the canonical energy is that, unlike the metrical one, it is sensitive to the addition of boundary terms to the action, as it happens to the AdS/CFT prescription Eq. (1).

Finally, from the AdS/CFT correspondence point of view, the proposal in [10] was to consider a generalized AdS/CFT prescription of the form (see also [15] for previous results)

$$
\exp \left(-I_{\mathrm{AdS}}\left[f_{0}\right]\right) \equiv\left\langle\exp \left(\int d^{d} x O(\vec{x}) f_{0}(\vec{x})\right)\right\rangle,
$$

where, unlike the usual prescription Eq. (1), we make use of a generalized source $f_{0}$ which depends on the boundary conditions. ${ }^{1}$ The formulation in [10] makes use of Dirichlet, Neumann, and mixed boundary conditions on the scalar field in both the minimally and nonminimally coupled cases. In addition, it involves a generalized Legendre transform prescription of the form

\footnotetext{
${ }^{1}$ Note that the addition of boundary terms to the action changes the boundary conditions under which the action is stationary, and on the other hand it also changes the generating functional for the boundary CFT. This is the reason why considering generalized boundary conditions as in Eq. (8) involves more information than making use of the usual prescription Eq. (1).
}

$$
\widetilde{I}_{\mathrm{AdS}}\left[f_{0}, \widetilde{f}_{0}\right]=I_{\mathrm{AdS}}\left[f_{0}\right]-\int d^{d} x f_{0}(\vec{x}) \widetilde{f}_{0}(\vec{x}),
$$

which differs from the usual one in [14] by the fact that it involves the whole on-shell action rather than only the leading nonlocal term. It was shown in [10] that the divergent local terms of the action contain information about the transformed generating functional, and then they have to be taken into account. In addition, the prescription above leaves no coefficient to be fixed "by hand." After performing the Legendre transformation, the transformed boundary CFT can be determined from the prescription

$$
\exp \left(-\widetilde{I}_{\mathrm{AdS}}\left[\widetilde{f}_{0}\right]\right) \equiv\left\langle\exp \left(\int d^{d} x \widetilde{O}(\vec{x}) \widetilde{f}_{0}(\vec{x})\right)\right\rangle .
$$

It was shown in [10] that this generalized Legendre transform prescription removes all problems mentioned above regarding the usual prescription. In addition, the key result in [10] has been to show that the generalized prescription Eqs. (8) -(10) gives rise to precisely the same constraints on $\varrho$ and $\nu$ for which irregular modes propagate in the bulk when the quantization is performed by making use of the canonical energy rather than the metrical one.

One of the purposes of this paper is to show that the introduction of double-trace perturbations at the boundary CFT can be understood in terms of the formulation in [10]. This would be a powerful result for many reasons. The first one is that it would lead in a natural way to a generalized AdS/CFT prescription in which multitrace interactions can be consistently incorporated.

The second reason is that it would enable us to relate the coupling coefficient of the double-trace perturbation to specific boundary conditions on the bulk field, or equivalently, to the addition of specific boundary terms to the action. In particular, we will show that there exist particular couplings which require us to consider new boundary conditions that have not been analyzed in [10]. In this article, we will also perform a detailed analysis of such new boundary conditions. In all cases, we will find the explicit form of the coupling coefficients and the surface terms.

The third reason is that to consider double-trace operators in terms of the formulation in [10] allows us to relate the corresponding coupling coefficients to the constraints on $\varrho$ and $\nu$ for which irregular modes propagate in the bulk when the canonical energy is employed instead of the metrical one. In particular, we will show that, when such constraints are satisfied, the couplings of the double-trace perturbations diverge. This result is consistent with the statement in [6] that, as the coupling grows, the system approaches the condition that is suitable for quantization to get a field of dimension $\Delta_{-}$. It is also important to point out that, in this formulation, the canonical energy in the bulk depends on the boundary multitrace perturbations.

And the last reason why it is useful to analyze doubletrace operators in terms of the formulation in [10] is that it allows us to extend, in a natural way, the formulation of double-trace perturbations to the nonminimally coupled case. In particular, an interesting result that we will find is that the 
introduction of a double-trace perturbation at the boundary generates the natural extension of the Gibbons-Hawking surface term [16], which is added to the Einstein-Hilbert action in order to have a well-defined variational principle under variations of the metric.

We also aim at connecting to the recently proposed formulation in [17] for an improved correspondence formula which gives correct boundary field theory correlators for multitrace perturbations. In particular, the key observation in $[6,17]$ is that the conformal operator $O$ is to be identified with the Legendre transformed field $\widetilde{\phi}_{0}$. In this paper, we claim that, in order to map to the boundary all the information contained in the bulk, we also need to identify, in the transformed formulation, the conjugated operator $\widetilde{O}$ with the field $\phi_{0}$. In particular, we will show that to require consistency imposes a precise constraint between the couplings of the double-trace perturbations corresponding to the conformal operators $O$ and $\widetilde{O}$. Another difference with respect to the formulation in [17] is that, in this work, we consider that the coupling of the double-trace perturbation depends on the distance to the boundary. We will show that this is needed for consistency. In particular, a result that we will find is that when the constraints for which irregular modes are allowed to propagate in the bulk are satisfied, the couplings of the double-trace perturbations corresponding to the operators $O$ and $\widetilde{O}$ have different asymptotic behaviors. A final thing to be mentioned is that, in this paper, we will consider the full functionals containing all local and nonlocal terms, unlike the formalism in [17], which considers only the leading nonlocal term. We do so because the divergent local terms contain information about the transformed generating functional, and then they have to be taken into account [10].

The paper is organized as follows. In Sec. II, we revisit the formulation in [17] and claim that, in order to map to the boundary all the information contained in the bulk, we also need to identify, in the transformed formulation, the conjugated operator $\widetilde{O}$ with the field $\phi_{0}$. We find the explicit form of the generating functionals for the boundary CFT's in terms of the couplings of the double-trace perturbations corresponding to the operators $O$ and $\widetilde{O}$. We also show that the requirement for consistency imposes a constraint between such couplings. In Sec. III, we analyze the precise way in which the introduction of double-trace perturbations changes the boundary conditions on the field by adding surface terms to the action, and extend the formulation to the nonminimally coupled case. In this situation, we will show that the introduction of double-trace perturbations generates the natural extension of the Gibbons-Hawking term. We also show how double-trace perturbations can be understood in terms of the generalized AdS/CFT prescription and the new quantization in the bulk as developed in [10]. In addition, an interesting result that we will find is that irregular modes are allowed to propagate precisely when the asymptotic behaviors of the couplings of the double-trace perturbations corresponding to the operators $O$ and $\widetilde{O}$ are different. In this situation, the coupling corresponding to the operator of conformal dimension $\Delta_{+}$diverges. We also show that a com- plete formulation requires the inclusion of new boundary conditions which have not been considered in [10]. We perform a detailed analysis of such new boundary conditions. Finally, Sec. IV contains our conclusions.

\section{DOUBLE-TRACE OPERATORS AND ADS/CFT}

Throughout this paper, we work in the Euclidean representation of the $\operatorname{AdS}_{d+1}$ in Poincare coordinates, described by the half space $x_{0}>0, x_{i} \in \mathbf{R}$ with the metric

$$
d s^{2}=\frac{1}{x_{0}^{2}} \sum_{\mu=0}^{d} d x^{\mu} d x^{\mu},
$$

where we have fixed the radius of $\operatorname{AdS}_{d+1}$ equal to 1 . We consider the space as foliated by a family of surfaces $x_{0}=\epsilon$ homeomorphic to the boundary at $x_{0}=0$. The corresponding outward pointing unit normal vector is

$$
n_{\mu}=\left(-\epsilon^{-1}, \mathbf{0}\right) \text {. }
$$

The limit $\epsilon \rightarrow 0$ is to be taken only at the end of calculations [18].

The usual action for the massive scalar field reads

$$
I_{0}=\frac{1}{2} \int d^{d+1} x \sqrt{g}\left(g^{\mu \nu} \partial_{\mu} \phi \partial_{\nu} \phi+M^{2} \phi^{2}\right)
$$

Performing an infinitesimal variation of the scalar field $\phi$ $\rightarrow \phi+\delta \phi$, the action above transforms as

$$
\delta I_{0}=\int d^{d} x \sqrt{h} \partial_{n} \phi_{\epsilon} \delta \phi_{\epsilon}
$$

where $h_{\mu \nu}$ is the induced metric at the surface $x_{0}=\epsilon, \phi_{\epsilon}$ is the value of the field at $x_{0}=\epsilon$, and $\partial_{n} \phi$ is the Lie derivative of $\phi$ along $n_{\mu}$. It is given by

$$
\partial_{n} \phi=n^{\mu} \partial_{\mu} \phi .
$$

Note that, in Eq. (14), the absence of a bulk contribution is due to the equation of motion

$$
\nabla^{2} \phi-M^{2} \phi=0 .
$$

The variation Eq. (14) shows that the action $I_{0}$ is stationary for a Dirichlet boundary condition which fixes the value of the scalar field $\phi$ at $x_{0}=\epsilon$, namely

$$
\delta \phi_{\epsilon}=0 .
$$

Integrating by parts and making use of the equation of motion, the action Eq. (13) can be written as the following pure-surface term:

$$
I_{0}=\frac{1}{2} \int d^{d} x \sqrt{h} \phi_{\epsilon} \partial_{n} \phi_{\epsilon} .
$$

The next step involves solving the equation of motion and writing $\partial_{n} \phi_{\epsilon}$ in terms of the boundary data $\phi_{\epsilon}$. This procedure has been carried out in $[18,19]$, where the final result 
was expanded in powers of the distance to the boundary in order to select the leading nonlocal term, which is understood as the generating functional for the boundary CFT [8]. However, the divergent local terms of the action contain information about the Legendre transformed generating functional [10], and then we need to take them into account. This means that, instead of the leading nonlocal term, we will make use of the full action containing all local and nonlocal terms. It reads

$$
\begin{aligned}
I_{0}\left[f_{\epsilon}\right]= & -\frac{1}{2} \int d^{d} x d^{d} y \sqrt{h} f_{\epsilon}(\vec{x}) f_{\epsilon}(\vec{y}) \\
& \times \int \frac{d^{d} k}{(2 \pi)^{d}} e^{-i \vec{k} \cdot(\vec{x}-\vec{y})} F(k \epsilon),
\end{aligned}
$$

where $f_{\epsilon}(\vec{x})$ is the source which couples to the boundary conformal operator through the prescription Eq. (1), $\vec{x}$ $=\left(x^{1}, \ldots, x^{d}\right), k=|\vec{k}|$ and

$$
F(k \epsilon)=\frac{d}{2}+\nu-k \epsilon \frac{K_{\nu+1}(k \epsilon)}{K_{\nu}(k \epsilon)} .
$$

Here $K_{\nu}$ is the modified Bessel function.

The action Eq. (19) is only one of the two functionals which contain the information about the boundary CFT's. The another one is obtained by performing the Legendre transformation Eq. (9), which gives rise to the following transformed functional containing all local and nonlocal terms $[10]$

$$
\begin{aligned}
\tilde{I}_{0}\left[\tilde{f}_{\epsilon}\right]= & \frac{1}{2} \int d^{d} x d^{d} y \sqrt{h} \tilde{f}_{\epsilon}(\vec{x}) \tilde{f}_{\epsilon}(\vec{y}) \\
& \times \int \frac{d^{d} k}{(2 \pi)^{d}} e^{-i \vec{k} \cdot(\vec{x}-\vec{y})} \frac{1}{F(k \epsilon)} .
\end{aligned}
$$

Both functionals $I_{0}$ and $\widetilde{I}_{0}$ are needed in order to map to the boundary all the information contained in the bulk. Each one of the sources $f_{\epsilon}$ and $\widetilde{f}_{\epsilon}$ will couple, after performing the limit $\epsilon \rightarrow 0$ in a proper way and through the prescriptions Eqs. (8) and (10), to the corresponding boundary conformal operator.

The important result in $[6,17]$ is that the transformed source $\widetilde{f}_{0}$ can be identified with the conformal operator $O$. In order to do this, we transform back the functional $\widetilde{I}_{0}\left[\tilde{f}_{\epsilon}\right]$ and find

$$
\begin{aligned}
I_{0}= & \int d^{d} x d^{d} y \sqrt{h} \int \frac{d^{d} k}{(2 \pi)^{d}} e^{-i \vec{k} \cdot(\vec{x}-\vec{y})} \\
& \times\left[\frac{1}{2} \frac{1}{F(k \epsilon)} \widetilde{f}_{\epsilon}(\vec{x}) \widetilde{f}_{\epsilon}(\vec{y})+f_{\epsilon}(\vec{x}) \tilde{f}_{\epsilon}(\vec{y})\right] .
\end{aligned}
$$

Now we will analyze the way in which the results above are affected by perturbing at the boundary with a double-trace operator of the form

$$
W[O]=\frac{\beta}{2} O^{2},
$$

where $\beta$ is the coupling coefficient. Following $[6,17]$, the next step is to identify $O$ with $\widetilde{f}_{0}$. This would give

$$
W\left[\widetilde{f}_{0}\right]=\frac{\beta}{2} \widetilde{f}_{0}^{2}
$$

However, it is important to note that the limit $\epsilon \rightarrow 0$ is to be taken only at the very end of calculations $[18] .^{2}$ This means that, instead of the above equation, we must consider

$$
W_{\epsilon}\left[\tilde{f}_{\epsilon}\right]=\frac{\beta(\epsilon)}{2} \widetilde{f}_{\epsilon}^{2},
$$

where we have replaced $\widetilde{f}_{0}$ with $\widetilde{f}_{\epsilon}$. Note that there is still another important difference between Eqs. (24), (25), namely that we have also introduced a dependence of the coupling $\beta$ on the distance to the boundary $\epsilon$. We will show that this is needed for consistency.

From the above considerations, we write the perturbed functional as

$$
\begin{aligned}
I= & \int d^{d} x d^{d} y \sqrt{h} \int \frac{d^{d} k}{(2 \pi)^{d}} e^{-i \vec{k} \cdot(\vec{x}-\vec{y})} \\
& \times\left[\frac{1}{2}\left(\frac{1}{F(k \epsilon)}+\beta(k \epsilon)\right) \tilde{f}_{\epsilon}(\vec{x}) \tilde{f}_{\epsilon}(\vec{y})+f_{\epsilon}(\vec{x}) \tilde{f}_{\epsilon}(\vec{y})\right],
\end{aligned}
$$

which for $\beta=0$ reduces to Eq. (22). The above expression is to be contrasted with the one in [17]. There are two important differences, namely that $\beta$ depends on $\epsilon$ and that $F(k \epsilon)$ contains all local and nonlocal terms, rather than only the leading nonlocal term. ${ }^{3}$

Setting $\partial I / \partial \widetilde{f}_{\epsilon}=0$, we get

$$
\widetilde{f}_{\epsilon}=-\frac{F(k \epsilon)}{1+\beta(k \epsilon) F(k \epsilon)} f_{\epsilon},
$$

and introducing this into Eq. (26) we find

\footnotetext{
${ }^{2}$ This acquires a new importance in the light of the result in [10] that the divergent local terms of the action contain information about the transformed generating functional. To take the limit $\epsilon$ $\rightarrow 0$ at this early stage would imply losing information.

${ }^{3}$ The formulation in [17] makes use, instead of $F(k \epsilon)$, of the conformal Green function $G(k)=-\{[\Gamma(1-\nu)] /[\Gamma(1+\nu)]\}$ $\times(k / 2)^{2 \nu}$, which involves a procedure in which the Legendre transformation is performed after having selected the leading nonlocal term, rather than following the opposite way as in [10].
} 


$$
\begin{aligned}
I\left[f_{\epsilon}\right]= & -\frac{1}{2} \int d^{d} x d^{d} y \sqrt{h} f_{\epsilon}(\vec{x}) f_{\epsilon}(\vec{y}) \\
& \times \int \frac{d^{d} k}{(2 \pi)^{d}} e^{-i \vec{k} \cdot(\vec{x}-\vec{y})} \frac{F(k \epsilon)}{1+\beta(k \epsilon) F(k \epsilon)} .
\end{aligned}
$$

Comparison with Eq. (19) shows that the double-trace perturbation Eq. (23) has introduced the replacement

$$
F(k \epsilon) \rightarrow \frac{F(k \epsilon)}{1+\beta(k \epsilon) F(k \epsilon)} .
$$

In the next section, we will show that this can be understood as a modification of the boundary conditions on the field, or equivalently, as the addition of a surface term to the action Eq. (13). We will also show how to extend the formulation to the case of a scalar field nonminimally coupled to the metric.

It has been stated in [17] that the above formulation applies to both regular and irregular modes propagating in the bulk. However, the formulation remains incomplete, because so far we have only considered a perturbation in the conformal operator $O$ [see Eq. (23)]. We still need to consider a perturbation in the conjugated operator $\widetilde{O}$, and we will show that the requirement for consistency imposes a precise constraint between both perturbations.

Note that, in the transformed situation, we identify $f_{0}$ with the conjugated operator $\widetilde{O}$. In order to do this, we perform a Legendre transform in Eq. (19) and get

$$
\begin{aligned}
\widetilde{I}_{0}= & -\int d^{d} x d^{d} y \sqrt{h} \int \frac{d^{d} k}{(2 \pi)^{d}} e^{-i \vec{k} \cdot(\vec{x}-\vec{y})} \\
& \times\left[\frac{1}{2} F(k \epsilon) f_{\epsilon}(\vec{x}) f_{\epsilon}(\vec{y})+\widetilde{f}_{\epsilon}(\vec{x}) f_{\epsilon}(\vec{y})\right] .
\end{aligned}
$$

We introduce the following double-trace perturbation in $\widetilde{O}$,

$$
\widetilde{W}[\widetilde{O}]=\frac{\widetilde{\beta}}{2} \widetilde{O}^{2},
$$

and, identifying $f_{0}$ with the conjugated operator $\widetilde{O}$, we write the perturbed functional as

$$
\begin{aligned}
\widetilde{I}= & -\int d^{d} x d^{d} y \sqrt{h} \int \frac{d^{d} k}{(2 \pi)^{d}} e^{-i \vec{k} \cdot(\vec{x}-\vec{y})} \\
& \times\left\{\frac{1}{2}[F(k \epsilon)-\widetilde{\beta}(k \epsilon)] f_{\epsilon}(\vec{x}) f_{\epsilon}(\vec{y})+\widetilde{f}_{\epsilon}(\vec{x}) f_{\epsilon}(\vec{y})\right\} .
\end{aligned}
$$

Note that, as we have done with the coupling $\beta$, we have introduced a dependence of $\widetilde{\beta}$ on $\epsilon$. Setting $\partial \widetilde{I} / \partial f_{\epsilon}=0$, we get

$$
f_{\epsilon}=-\frac{1}{F(k \epsilon)-\widetilde{\beta}(k \epsilon)} \widetilde{f}_{\epsilon},
$$

and introducing this into Eq. (32) we find

$$
\begin{aligned}
\widetilde{I}\left[\widetilde{f}_{\epsilon}\right]= & \frac{1}{2} \int d^{d} x d^{d} y \sqrt{h} \widetilde{f}_{\epsilon}(\vec{x}) \widetilde{f}_{\epsilon}(\vec{y}) \\
& \times \int \frac{d^{d} k}{(2 \pi)^{d}} e^{-i \vec{k} \cdot(\vec{x}-\vec{y})} \frac{1}{F(k \epsilon)-\widetilde{\beta}(k \epsilon)} .
\end{aligned}
$$

There is still one last thing to be considered in order to have a complete and consistent formulation, and it is to require the expression above to be actually the Legendre transform of Eq. (28). As anticipated, this will impose a precise constraint between $\beta$ and $\widetilde{\beta}$. By Legendre transforming Eq. (28), we find

$$
\begin{aligned}
\widetilde{I}\left[\tilde{f}_{\epsilon}\right]= & \frac{1}{2} \int d^{d} x d^{d} y \sqrt{h} \widetilde{f}_{\epsilon}(\vec{x}) \tilde{f}_{\epsilon}(\vec{y}) \\
& \times \int \frac{d^{d} k}{(2 \pi)^{d}} e^{-i \vec{k} \cdot(\vec{x}-\vec{y})} \frac{1+\beta(k \epsilon) F(k \epsilon)}{F(k \epsilon)} .
\end{aligned}
$$

From Eqs. (34),(35) we find the constraint

$$
\widetilde{\beta}(k \epsilon)=\frac{\beta(k \epsilon) F^{2}(k \epsilon)}{1+\beta(k \epsilon) F(k \epsilon)},
$$

which is required for consistency. Note that both functionals Eqs. (28),(35) are needed in order to map to the boundary all the information contained in the bulk. The corresponding boundary CFT's can be obtained through the prescriptions Eqs. (8), (10). We emphasize that the functionals Eqs. (28), (35) contain all local and nonlocal terms, and that this is needed in order to map to the boundary the constraints that the quantization imposes in the bulk [10]. This topic will be discussed in the following section.

So far, we have developed a generic formulation in which the double-trace perturbations at the boundary of $\mathrm{AdS}_{d+1}$ can be consistently incorporated into an extended AdS/CFT prescription. We still need to understand the precise way in which such perturbations change the boundary conditions on the field by adding surface terms to the action Eq. (13), and to extend the formalism to the nonminimally coupled case. This is left for the following section, where we will connect the formulation developed so far to the generalized AdS/CFT prescription in [10], which analyzes the role of boundary conditions in the AdS/CFT correspondence. We will also relate the previous formalism to the energy of the theory on the bulk, and to the existence of constraints for which the irregular modes are allowed to propagate. We will find the explicit expressions of the couplings, and show that they exhibit very interesting behaviors when irregular modes are also allowed to propagate.

\section{GENERALIZED BOUNDARY CONDITIONS}

In this section, we will show that the double-trace perturbations at the boundary of $\operatorname{AdS}_{d+1}$ can be understood as the introduction of Dirichlet, Neumann, and mixed boundary conditions on the scalar field. We will consider both mini- 
mally and nonminimally coupled cases. We begin by concentrating on the minimally coupled case.

\section{A. The minimally coupled case}

In this case, we set $\varrho=0$ in Eq. (7), thus getting $\nu$ $=\sqrt{\left(d^{2} / 4\right)+m^{2}}$. It is natural to begin by analyzing the simplest case in which the coupling $\beta$ does not depend on the distance to the boundary. For this purpose, we set

$$
\beta(k \epsilon)=-2 \lambda,
$$

where $\lambda$ is a real coefficient. Then, from Eq. (36) we also find

$$
\widetilde{\beta}(k \epsilon)=-\frac{2 \lambda F^{2}(k \epsilon)}{1-2 \lambda F(k \epsilon)} .
$$

Note, then, that consistency of the formulation requires that at least one of the couplings depends on the distance to the boundary, as anticipated in the previous section. We will later show that the particular cases for which irregular modes are also allowed to propagate in the bulk correspond to the situations in which $\beta$ and $\widetilde{\beta}$ have different asymptotic behaviors.

Under the identification Eq. (37), the functionals Eqs. (28),(35) read

$$
\begin{aligned}
I\left[f_{\epsilon}\right]= & -\frac{1}{2} \int d^{d} x d^{d} y \sqrt{h} f_{\epsilon}(\vec{x}) f_{\epsilon}(\vec{y}) \\
& \times \int \frac{d^{d} k}{(2 \pi)^{d}} e^{-i \vec{k} \cdot(\vec{x}-\vec{y})} \frac{F(k \epsilon)}{1-2 \lambda F(k \epsilon)}, \\
\widetilde{I}\left[\tilde{f}_{\epsilon}\right]= & \frac{1}{2} \int d^{d} x d^{d} y \sqrt{h} \widetilde{f}_{\epsilon}(\vec{x}) \tilde{f}_{\epsilon}(\vec{y}) \\
& \times \int \frac{d^{d} k}{(2 \pi)^{d}} e^{-i \vec{k} \cdot(\vec{x}-\vec{y})} \frac{1-2 \lambda F(k \epsilon)}{F(k \epsilon)} .
\end{aligned}
$$

The key result is that the above functionals are precisely the ones found in [10] when considering a boundary condition which fixes at the border the field

$$
\phi+2 \lambda \partial_{n} \phi .
$$

Then, for the particular choice Eq. (37), the double-trace perturbation acts by turning the usual Dirichlet boundary condition into a mixed one. ${ }^{4}$ This is also equivalent to adding to the action Eq. (13) a boundary term of the form [10]

$$
I=I_{0}+\lambda \int d^{d} x \sqrt{h}\left(\partial_{n} \phi_{\epsilon}\right)^{2} .
$$

Note that, in the particular case $\lambda=0$, we recover the usual Dirichlet boundary condition. One important point is that for general $\lambda$ both functionals Eqs. (39),(40) correspond to the

\footnotetext{
${ }^{4}$ In [10], this particular boundary condition was called the "type II" mixed boundary condition.
}

same boundary conformal dimension, namely $\Delta_{+}$. However, in the particular situation in which both constraints [10]

$$
\lambda=\frac{1}{2 \Delta_{-}}
$$

and

$$
\nu<1
$$

are satisfied, the divergent local terms of both functionals Eqs. (39),(40) cancel out. This fact encodes the information that the Legendre transform interpolates between different conformal dimensions, namely $\Delta_{+}$and $\Delta_{-}$. In this situation, the addition of counterterms is not required. From the bulk point of view, in this case both regular and irregular modes can propagate, because the canonical energy is conserved, positive, and finite for both of them. When Eq. (43) is satisfied, but Eq. (44) is not, the conformal dimension $\Delta_{-}$ reaches the unitarity bound $(d-2) / 2$, and becomes independent of the mass. The unitarity bound is also reached for $m$ $=0$. For details, see [10].

We will now show that there exists an interesting relation between the values and asymptotic behaviors of $\beta$ and $\widetilde{\beta}$ and the phenomenon of the propagation of irregular modes in the bulk. Let us first consider the situation in which Eq. (43) is not satisfied. For simplicity, we concentrate on the case of $\nu$ not an integer. ${ }^{5}$ By expanding the Bessel functions in Eq. (38) in powers of $\epsilon$, we find

$$
\widetilde{\beta}(k \epsilon)=-\frac{2 \lambda \Delta_{-}^{2}}{1-2 \lambda \Delta_{-}}+\cdots,
$$

where the dots stand for higher-order terms in $\epsilon$. Comparison with Eq. (37) shows that in this case, where only regular modes are allowed to propagate in the bulk, $\beta$ and $\widetilde{\beta}$ have the same asymptotic behavior, namely, both of them differ from a constant only on terms which vanish as we approach the boundary. We also note from Eq. (45) that $\widetilde{\beta}$ diverges as we get closer to the critical value Eq. (43) for which irregular modes are also allowed to propagate. Note that this result is consistent with the statement in [6] that, as the coupling grows, the system approaches the condition that is suitable for quantization to get a field of dimension $\Delta_{-}$.

We finally consider the situation where Eq. (43) is satisfied and irregular modes are allowed to propagate as well. We expect from the above results that something special will happen in this particular situation. We will show that, in fact, in this case $\beta$ and $\widetilde{\beta}$ have different asymptotic behaviors, and the last one diverges as we approach the boundary. We have to consider separately the cases where Eq. (44) is, or is not, also satisfied. Expanding in Eq. (38) we find, for $\nu<1$,

\footnotetext{
${ }^{5}$ The case of $\nu$ integer is analogous. The only difference is that there arise additional logarithmic terms which must be taken into account (see, for example, [15]).
} 


$$
\widetilde{\beta}(k \epsilon)=-2^{2 \nu-1} \Delta_{-}^{2} \frac{\Gamma(\nu)}{\Gamma(1-\nu)}(k \epsilon)^{-2 \nu}+\cdots,
$$

whereas for $\nu>1$ we get

$$
\widetilde{\beta}(k \epsilon)=-2(\nu-1) \Delta_{-}^{2}(k \epsilon)^{-2}+\cdots .
$$

In the above equations, the dots stand for higher-order terms in $\epsilon$. As anticipated, we find that, in the particular situation where irregular modes are also allowed to propagate in the bulk, the couplings $\beta$ and $\widetilde{\beta}$ have different asymptotic behaviors, and the last one, which corresponds to the operator of conformal dimension $\Delta_{+}$[10], diverges as we approach the boundary.

So far, we have considered the situation where $\beta$ does not depend on the distance to the boundary. As the next step, it is natural to analyze the case where $\beta$ depends on $\epsilon$, and $\widetilde{\beta}$ does not. In order to do this, we consider, instead of Eq. (37), the following starting point:

$$
\widetilde{\beta}(k \epsilon)=-2 \lambda,
$$

where, as before, $\lambda$ is a real coefficient. From Eq. (36), we get

$$
\beta(k \epsilon)=-\frac{2 \lambda}{F(k \epsilon)} \frac{1}{F(k \epsilon)+2 \lambda} .
$$

From the above equation, we write the functionals Eqs. (28),(35) as

$$
\begin{aligned}
I\left[f_{\epsilon}\right]= & -\frac{1}{2} \int d^{d} x d^{d} y \sqrt{h} f_{\epsilon}(\vec{x}) f_{\epsilon}(\vec{y}) \\
& \times \int \frac{d^{d} k}{(2 \pi)^{d}} e^{-i \vec{k} \cdot(\vec{x}-\vec{y})}[F(k \epsilon)+2 \lambda], \\
\widetilde{I}\left[\widetilde{f}_{\epsilon}\right]= & \frac{1}{2} \int d^{d} x d^{d} y \sqrt{h} \tilde{f}_{\epsilon}(\vec{x}) \tilde{f}_{\epsilon}(\vec{y}) \\
& \times \int \frac{d^{d} k}{(2 \pi)^{d}} e^{-i \vec{k} \cdot(\vec{x}-\vec{y})} \frac{1}{F(k \epsilon)+2 \lambda} .
\end{aligned}
$$

The functionals above have not been considered in [10]. However, by following an analogous procedure, it can be verified that they correspond to considering a Dirichlet boundary condition and adding to the usual action Eq. (13) a boundary term of the form

$$
I=I_{0}-\lambda \int d^{d} x \sqrt{h} \phi_{\epsilon}^{2} .
$$

The fact that the above action is stationary under a Dirichlet boundary condition can be verified by noting that, under a transformation $\phi \rightarrow \phi+\delta \phi$, its variation is given by the following surface term:

$$
\delta I=\int d^{d} x \sqrt{h}\left(\partial_{n} \phi_{\epsilon}-2 \lambda \phi_{\epsilon}\right) \delta \phi_{\epsilon},
$$

which vanishes for $\delta \phi_{\epsilon}=0$. Since we are still considering a Dirichlet boundary condition, it may seem that in this situation the double-trace perturbation causes no effect. However, this is not the case, because the importance of the surface term in Eq. (52) is that it allows for irregular modes to propagate in the bulk for particular values of $\lambda$. This can be verified by making use of a formalism which is analogous to the one developed in [10] for other possible boundary conditions. In particular, it can be seen that for

$$
\lambda=-\frac{\Delta_{-}}{2},
$$

the canonical energy is conserved, positive, and finite for both regular and irregular modes propagating in the bulk, provided that the constraint Eq. (44) is satisfied as well. ${ }^{6} \mathrm{We}$ emphasize that, when both constraints Eqs. (44),(54) are satisfied, the Legendre transform interpolates between different conformal dimensions $\Delta_{+}$and $\Delta_{-}$, due to the cancellation of the divergent local terms of the functionals Eqs. (50),(51). This statement can be verified by following a procedure analogous to the one in [10]. When Eq. (54) is satisfied, but Eq. (44) is not, the conformal dimension $\Delta_{-}$reaches the unitarity bound $(d-2) / 2$.

Now we analyze the existing relation between the values and asymptotic behaviors of $\beta$ and $\widetilde{\beta}$ and the phenomenon of the propagation of irregular modes. We have seen that in the previously analyzed mixed boundary condition, the coupling $\widetilde{\beta}$ diverges when the critical value Eq. (43) is reached. Since in the current Dirichlet situation the conformal dimension $\Delta_{-}$corresponds to the conformal operator $\widetilde{O}$, rather than to $O,{ }^{7}$ then in this situation we expect that it will be $\beta$, rather than $\widetilde{\beta}$, the coupling which diverges as we approach the critical value Eq. (54). We now verify this statement. Again, we concentrate on the case of $\nu$ not an integer. Let us first consider the situation when Eq. (54) is not satisfied. Expanding in $\epsilon$ in Eq. (49), we find

$$
\beta(k \epsilon)=-\frac{2 \lambda}{\Delta_{-}\left(\Delta_{-}+2 \lambda\right)}+\cdots,
$$

\footnotetext{
${ }^{6}$ Since this particular case has not been analyzed in [10], we summarize here the main results. Making use of global coordinates $(\tau, \rho, \vec{\Omega})$ defined as in [10] (recall here that $\tau$ is the time coordinate, $\rho$ is the radial coordinate, and $\vec{\Omega}$ are the angular coordinates) we find from the action Eq. (52) that the canonical energy is of the form $E=-\int d^{d} x \sqrt{g}\left[\Theta_{\tau}^{\tau}+\lambda \nabla_{\mu}\left(n^{\mu} \phi^{2}\right)\right]$, where the integration is carried out over the spatial coordinates, and $\Theta_{\mu \nu}=\partial_{\mu} \phi \partial_{\nu} \phi$ $-\frac{1}{2} g_{\mu \nu}\left[g^{\alpha \beta} \partial_{\alpha} \phi \partial_{\beta} \phi+m^{2} \phi^{2}\right]$. It can be verified that, for regular modes propagating in the bulk, the canonical energy is conserved, positive, and finite for any values of $\lambda$ and $\nu$, whereas for irregular modes propagating in the bulk the canonical energy is conserved, positive, and finite only when the constraints Eqs. (44),(54) are satisfied.

${ }^{7}$ This result can be found by computing the boundary CFT's through the prescription Eqs. (8),(10).
} 
where the dots stand for higher-order terms in $\epsilon$. We note from Eq. (48) that, as in the previous mixed case, $\beta$ and $\widetilde{\beta}$ have the same asymptotic behavior when only regular modes propagate in the bulk. Note also that, as expected, $\beta$ diverges as we approach the critical value Eq. (54) for which irregular modes are allowed to propagate as well.

Now we analyze the case when the constraint Eq. (54) is satisfied. The calculations are analogous to the ones performed in the previous mixed case, and the results are as follows. For $\nu<1$ we get

$$
\beta(k \epsilon)=2^{2 \nu} \frac{\lambda}{\Delta_{-}} \frac{\Gamma(\nu)}{\Gamma(1-\nu)}(k \epsilon)^{-2 \nu}+\cdots,
$$

whereas for $\nu>1$ we find

$$
\beta(k \epsilon)=4(\nu-1) \frac{\lambda}{\Delta_{-}}(k \epsilon)^{-2}+\cdots .
$$

Then, as in the previous mixed case, in the situation when the irregular modes are also allowed to propagate the couplings have different asymptotic behaviors, as noticed by comparing Eqs. (56),(57) to Eq. (48). We also note that in this case the coupling $\beta$, which corresponds to the conformal operator of dimension $\Delta_{+}$, diverges as we approach the boundary.

So far, we have considered the situations in which one of the couplings does not depend on $\epsilon$. Since the consistency relation Eq. (36) makes it impossible to consider a case where both couplings are simultaneously independent of the distance to the boundary, there is only one case left to be considered, namely the situation in which both couplings depend on $\epsilon$. In order to analyze this case, we set

$$
\beta(k \epsilon)=-\frac{1+F(k \epsilon)[F(k \epsilon)-2 \lambda]}{F(k \epsilon)},
$$

and from Eq. (36) we also find

$$
\widetilde{\beta}(k \epsilon)=\frac{1+F(k \epsilon)[F(k \epsilon)-2 \lambda]}{F(k \epsilon)-2 \lambda} .
$$

The reason why we choose the particular expressions above will be clarified later. Inserting Eq. (58) into Eqs. (28),(35), we get

$$
\begin{aligned}
I\left[f_{\epsilon}\right]= & \frac{1}{2} \int d^{d} x d^{d} y \sqrt{h} f_{\epsilon}(\vec{x}) f_{\epsilon}(\vec{y}) \\
& \times \int \frac{d^{d} k}{(2 \pi)^{d}} e^{-i \vec{k} \cdot(\vec{x}-\vec{y})} \frac{1}{F(k \epsilon)-2 \lambda}, \\
\widetilde{I}\left[\widetilde{f}_{\epsilon}\right]= & -\frac{1}{2} \int d^{d} x d^{d} y \sqrt{h} \widetilde{f}_{\epsilon}(\vec{x}) \tilde{f}_{\epsilon}(\vec{y}) \\
& \times \int \frac{d^{d} k}{(2 \pi)^{d}} e^{-i \vec{k} \cdot(\vec{x}-\vec{y})}[F(k \epsilon)-2 \lambda] .
\end{aligned}
$$

The key result is that, as can be verified, the above functionals correspond to adding to the action Eq. (13) the following surface terms:

$$
I=I_{0}-\lambda \int d^{d} x \sqrt{h} \phi_{\epsilon}^{2}-\int d^{d} x \sqrt{h} \phi_{\epsilon} \partial_{n} \phi_{\epsilon},
$$

which give rise to an action which is stationary under a boundary condition which fixes at the border the field ${ }^{8}$

$$
\partial_{n} \phi+2 \lambda \phi .
$$

This means that the double-trace perturbation Eq. (58) turns the usual Dirichlet boundary condition into a mixed one which is different from the previously analyzed mixed boundary condition [see Eq. (41)]. This clarifies the reason why we chose the specific coupling Eq. (58). Different choices may not make sense in terms of a problem of boundary conditions on the scalar field. Note that, in the particular case $\lambda=0$, this new mixed boundary condition reduces to a Neumann one. Even when this boundary condition has not been analyzed in [10], the procedure is analogous, and it can be verified that in this situation the irregular modes are allowed to propagate in the bulk as well as the regular ones only when the constraint ${ }^{9}$

$$
\lambda=\frac{\Delta_{-}}{2}
$$

is satisfied together with Eq. (44). It can be shown that in this situation the divergent local terms of the functionals Eqs. (60),(61) cancel out and, correspondingly, the Legendre transform interpolates between different conformal dimensions, namely $\Delta_{+}$and $\Delta_{-}$. When Eq. (64) is satisfied, but Eq. (44) is not, the conformal dimension $\Delta_{-}$reaches the unitarity bound $(d-2) / 2$.

Regarding the relation between the values and asymptotic behaviors of $\beta$ and $\widetilde{\beta}$ and the phenomenon of the propagation of irregular modes, we expect to find results which are analogous to the ones found in the previous cases. In particular, we expect that in this situation the coupling $\widetilde{\beta}$, which is the one that corresponds to the conformal operator of dimension $\Delta_{+},{ }^{10}$ should diverge as we approach the critical value

\footnotetext{
${ }^{8}$ Note that, under a transformation $\phi \rightarrow \phi+\delta \phi$, the variation of the action Eq. (62) is given by the surface term $\delta I=$ $-\int d^{d} x \sqrt{h} \phi_{\epsilon} \delta\left(\partial_{n} \phi_{\epsilon}+2 \lambda \phi_{\epsilon}\right)$, which vanishes under a boundary condition which fixes at the border the field Eq. (63).

${ }^{9}$ Since this particular case has not been analyzed in [10], we give here the main results. The canonical energy corresponding to the action Eq. (62) is given by $E=-\int d^{d} x \sqrt{g}\left[\Theta_{\tau}^{\tau}+\lambda \nabla_{\mu}\left(n^{\mu} \phi^{2}\right)\right.$ $\left.+\frac{1}{2}\left(\nabla^{2} \phi^{2}-\partial^{\tau} \partial_{\tau} \phi^{2}\right)\right]$, where we consider global coordinates $(\tau, \rho, \vec{\Omega})$ defined as in [10], the integration is carried out over the spatial coordinates, and $\Theta_{\mu \nu}=\partial_{\mu} \phi \partial_{\nu} \phi-\frac{1}{2} g_{\mu \nu}\left[g^{\alpha \beta} \partial_{\alpha} \phi \partial_{\beta} \phi\right.$ $+m^{2} \phi^{2}$. It can be shown that the canonical energy is conserved, positive, and finite for irregular modes propagating in the bulk only when both constraints Eqs. (44),(64) are satisfied.

${ }^{10}$ This result can be found by computing the boundary CFT's through the prescription Eqs. (8),(10).
} 
Eq. (64). Now we verify this statement for the case of $\nu$ not an integer. Since calculations are analogous to the ones performed in the previous cases, we just present the main results. Let us first consider that Eq. (64) is not satisfied. Then, expanding in Eqs. (58),(59), we find

$$
\begin{aligned}
& \beta(k \epsilon)=-\frac{1}{\Delta_{-}}\left[1+\Delta_{-}\left(\Delta_{-}-2 \lambda\right)\right]+\cdots, \\
& \widetilde{\beta}(k \epsilon)=\frac{1}{\Delta_{-}-2 \lambda}\left[1+\Delta_{-}\left(\Delta_{-}-2 \lambda\right)\right]+\cdots,
\end{aligned}
$$

where, as in the previous cases, the dots stand for higherorder terms in $\epsilon$. As anticipated, we note that $\beta$ and $\widetilde{\beta}$ have the same asymptotic behavior, and that $\widetilde{\beta}$ diverges as we approach the critical value Eq. (64).

Finally, we consider the situation in which the constraint Eq. (64) is satisfied. For $\nu<1$ we find

$$
\begin{aligned}
\beta(k \epsilon)= & -\frac{1}{\Delta_{-}}+\cdots, \\
\widetilde{\beta}(k \epsilon)= & -2^{2 \nu-1} \frac{\Gamma(\nu)}{\Gamma(1-\nu)}(k \epsilon)^{-2 \nu} \\
& +\cdots
\end{aligned}
$$

whereas for $\nu>1$ we get

$$
\begin{aligned}
& \beta(k \epsilon)=-\frac{1}{\Delta_{-}}+\cdots, \\
& \widetilde{\beta}(k \epsilon)=-2(\nu-1)(k \epsilon)^{-2}+\cdots .
\end{aligned}
$$

Then, as expected, the couplings have different asymptotic behaviors, and $\widetilde{\beta}$ diverges as we approach the boundary.

\section{B. The nonminimally coupled case}

In the previous subsection, we have shown, for the particular minimally coupled case, that the perturbation of the conformal field theory by a double-trace operator can be understood as the introduction of a generalized boundary condition on the scalar field. Now we consider the more general situation of a nonminimally coupled scalar field. In this case, we have a nonvanishing coupling coefficient $\varrho$ in Eq. (7). But, as pointed out in [10], the effect of the nonminimal coupling does not limit itself to a redefinition of the effective mass of the theory. In this subsection we will show, in particular, that the introduction of a double-trace perturbation at the boundary generates the natural extension of the GibbonsHawking surface term [16], which is added to the EinsteinHilbert action in order to have a well-defined variational principle under variations of the metric. As in the minimally coupled case, we will consider different boundary conditions on the scalar field and perform a detailed analysis of each one of them.

We begin by considering the simplest case where the coupling $\widetilde{\beta}$ does not depend on the distance to the boundary,

$$
\widetilde{\beta}(k \epsilon)=2 \varrho d,
$$

where $\varrho$ has been introduced in Eq. (7). Recall here that, throughout this section, we will consider $\nu$ $=\sqrt{\left(d^{2} / 4\right)+m^{2}+\varrho R}$. From Eq. (36), we also find

$$
\beta(k \epsilon)=\frac{2 \varrho d}{F(k \epsilon)} \frac{1}{F(k \epsilon)-2 \varrho d} .
$$

Inserting the above equation into Eqs. (28),(35), we get

$$
\begin{aligned}
I\left[f_{\epsilon}\right]= & -\frac{1}{2} \int d^{d} x d^{d} y \sqrt{h} f_{\epsilon}(\vec{x}) f_{\epsilon}(\vec{y}) \\
& \times \int \frac{d^{d} k}{(2 \pi)^{d}} e^{-i \vec{k} \cdot(\vec{x}-\vec{y})}[F(k \epsilon)-2 \varrho d], \\
\widetilde{I}\left[\widetilde{f}_{\epsilon}\right]= & \frac{1}{2} \int d^{d} x d^{d} y \sqrt{h} \widetilde{f}_{\epsilon}(\vec{x}) \tilde{f}_{\epsilon}(\vec{y}) \\
& \times \int \frac{d^{d} k}{(2 \pi)^{d}} e^{-i \vec{k} \cdot(\vec{x}-\vec{y})} \frac{1}{F(k \epsilon)-2 \varrho d} .
\end{aligned}
$$

The above functionals are precisely the same as the ones found in [10] when considering, in the nonminimally coupled case, a Dirichlet boundary condition which fixes the field $\phi$ at the border. We note from [10] that the above functionals correspond to adding to the usual action Eq. (13) a boundary term of the form

$$
I=I_{0}-\varrho \int d^{d} x \sqrt{h} \mathcal{K}_{\epsilon} \phi_{\epsilon}^{2}
$$

where $\mathcal{K}$ is the trace of the extrinsic curvature at the boundary. The above surface term is just the natural extension of the usual Gibbons-Hawking term [16]. The importance of this surface term is that, even when we are considering a Dirichlet boundary condition, it allows irregular modes to propagate in the bulk for particular values of $\varrho$ [10]. We have just shown that such a surface term can be generated by perturbing the conformal field theory with a double-trace operator. Note that in the particular case $\varrho=0$, corresponding to the minimally coupled case, we recover the usual Dirichlet boundary condition. We also point out that for general $\varrho$ both functionals Eqs. (73),(74) give rise to the same conformal dimension $\Delta_{+}$, with one particular exception, namely the situation in which both constraints Eq. (44) and [10]

$$
\varrho=\frac{d-1}{8 d}\left[1 \pm \sqrt{1+\left(\frac{4 m}{d-1}\right)^{2}}\right]
$$

are satisfied. ${ }^{11}$ In this case, the divergent local terms of both functionals Eqs. (73),(74) cancel out, making the addition of counterterms unnecessary, and the Legendre transform inter-

\footnotetext{
${ }^{11}$ It is interesting to note that, in the particular case $m=0$, one of the solutions in Eq. (76) vanishes, whereas the another one reduces to the conformal value for which the "improved" stress-energy tensor of the scalar field becomes traceless. This means that, in particular, Weyl-invariant theories in the bulk allow for irregular modes to propagate as well, and give rise to functionals in which the divergent local terms cancel out.
} 
polates between different conformal dimensions, namely $\Delta_{+}$ and $\Delta_{-}$. From the bulk point of view, both regular and irregular modes can propagate, because the canonical energy is conserved, positive, and finite for both of them. When Eq. (76) is satisfied but Eq. (44) is not, the conformal dimension $\Delta_{-}$reaches the unitarity bound $(d-2) / 2$. For details, see [10].

Now we concentrate on the relation between the values and asymptotic behaviors of $\beta$ and $\widetilde{\beta}$ and the phenomenon of the propagation of irregular modes in the bulk. Note that, in this case, the conformal dimension $\Delta_{-}$corresponds to the conformal operator $\widetilde{O}[10]$, and we expect $\beta$ to diverge as we approach any of the critical values Eq. (76). We begin by considering the situation in which Eq. (76) is not an satisfied. Expanding for $\nu$ not integer in Eq. (72), we find

$$
\beta(k \epsilon)=\frac{2 \varrho d}{\Delta_{-}\left(\Delta_{-}-2 \varrho d\right)}+\cdots,
$$

where the dots stand for higher-order terms in $\epsilon$. From Eqs. (71),(77), we find that $\beta$ and $\widetilde{\beta}$ have the same asymptotic behavior, as expected. Also as expected, we note from the above equation that $\beta$ diverges as we approach any of the critical values Eq. (76). ${ }^{12}$

We still need to consider the case when Eq. (76) is satisfied. This is done as in the previous cases. For $\nu<1$, we get

$$
\beta(k \epsilon)=-2^{2 \nu} \frac{\varrho d}{\Delta_{-}} \frac{\Gamma(\nu)}{\Gamma(1-\nu)}(k \epsilon)^{-2 \nu}+\cdots,
$$

whereas for $\nu>1$ we find

$$
\beta(k \epsilon)=-4(\nu-1) \frac{\varrho d}{\Delta_{-}}(k \epsilon)^{-2}+\cdots .
$$

As expected, if irregular modes are also allowed to propagate, then the couplings have different asymptotic behaviors, as noticed from Eqs. (71),(78), and (79). In this situation, $\beta$ diverges as we approach the boundary.

Finally, we consider a situation in which both couplings $\beta$ and $\widetilde{\beta}$ depend on the distance to the boundary. This is done by setting

$$
\beta(k \epsilon)=-\frac{1+F(k \epsilon)[F(k \epsilon)+2 \varrho d]}{F(k \epsilon)} .
$$

From Eq. (36), we also get

$$
\widetilde{\beta}(k \epsilon)=\frac{1+F(k \epsilon)[F(k \epsilon)+2 \varrho d]}{F(k \epsilon)+2 \varrho d} .
$$

Introducing Eq. (80) into Eqs. (28),(35), we find

\footnotetext{
${ }^{12}$ Recall here that $\Delta_{-}$depends on $\varrho$.
}

$$
\begin{aligned}
I\left[f_{\epsilon}\right]= & \frac{1}{2} \int d^{d} x d^{d} y \sqrt{h} f_{\epsilon}(\vec{x}) f_{\epsilon}(\vec{y}) \\
& \times \int \frac{d^{d} k}{(2 \pi)^{d}} e^{-i \vec{k} \cdot(\vec{x}-\vec{y})} \frac{1}{F(k \epsilon)+2 \varrho d} \\
\widetilde{I}\left[\tilde{f}_{\epsilon}\right]= & -\frac{1}{2} \int d^{d} x d^{d} y \sqrt{h} \widetilde{f}_{\epsilon}(\vec{x}) \tilde{f}_{\epsilon}(\vec{y}) \\
& \times \int \frac{d^{d} k}{(2 \pi)^{d}} e^{-i \vec{k} \cdot(\vec{x}-\vec{y})}[F(k \epsilon)+2 \varrho d]
\end{aligned}
$$

The key result is that the above functionals are precisely the same as the ones considered in [10] when analyzing a boundary condition which fixes at the boundary the field

$$
\partial_{n} \phi+2 \varrho \mathcal{K} \phi
$$

This means that, for the particular choice Eq. (80), the double-trace perturbation acts by turning the Dirichlet boundary condition into a mixed one, which is different from the ones considered in the minimally coupled case [see Eqs. $(41),(63)] .{ }^{13}$ It is also interesting to note from Eq. (84) that, in the particular minimally coupled case $(\varrho=0)$, this mixed boundary condition reduces to a Neumann one. We also point out that setting this mixed boundary condition is equivalent to adding to the usual action Eq. (13) the following boundary terms [10]:

$$
I=I_{0}-\varrho \int d^{d} x \sqrt{h} \mathcal{K}_{\epsilon} \phi_{\epsilon}^{2}-\int d^{d} x \sqrt{h} \phi_{\epsilon} \partial_{n} \phi_{\epsilon},
$$

where the first surface term is the same as the one that arises in the Dirichlet situation Eq. (75), whereas the second surface term is new. We emphasize that the new surface term does not spoil the property of having a well-defined variational principle under variations of the metric. We also point out that for general $\varrho$ both functionals Eqs. (82),(83) give rise to the same conformal dimension $\Delta_{+}$, with one particular exception, namely the situation in which both constraints Eq. (44) and [10]

$$
\varrho=-\frac{3 d+1}{8 d}\left[1 \mp \sqrt{1+\left(\frac{4 m}{3 d+1}\right)^{2}}\right]
$$

are satisfied. In analogy to the former cases, in this situation the divergent local terms of the functionals Eqs. (82),(83) cancel out, and this fact encodes the information that the Legendre transform interpolates between different conformal dimensions $\Delta_{+}$and $\Delta_{-}$. In this case, there is no need to add any counterterms. From the bulk point of view, both regular and irregular modes are allowed to propagate, because the canonical energy is conserved, positive, and finite for both of them. In addition, when Eq. (86) is satisfied but Eq. (44) is

\footnotetext{
${ }^{13}$ In [10], this particular boundary condition was called the "type I" mixed boundary condition.
} 
not, the conformal dimension $\Delta_{-}$reaches the unitarity bound $(d-2) / 2$ and becomes independent of the effective mass. For details, see [10].

We still need to analyze the relation between the values and asymptotic behaviors of $\beta$ and $\widetilde{\beta}$ and the phenomenon of the propagation of irregular modes. In this particular case, we expect $\widetilde{\beta}$ to diverge as we approach any of the critical values Eq. (86). This is due to the fact that $\widetilde{\beta}$ corresponds to the conformal operator of dimension $\Delta_{+}$[10]. Let us first consider the situation where Eq. (86) is not satisfied. Expanding in Eqs. (80),(81), we get

$$
\begin{aligned}
& \beta(k \epsilon)=-\frac{1}{\Delta_{-}}\left[1+\Delta_{-}\left(\Delta_{-}+2 \varrho d\right)\right]+\cdots, \\
& \widetilde{\beta}(k \epsilon)=\frac{1}{\Delta_{-}+2 \varrho d}\left[1+\Delta_{-}\left(\Delta_{-}+2 \varrho d\right)\right]+\cdots .
\end{aligned}
$$

As expected, $\beta$ and $\widetilde{\beta}$ have the same asymptotic behavior, and $\widetilde{\beta}$ diverges as we approach any of the critical values Eq. (86). ${ }^{14}$

Finally, we consider the situation in which Eq. (86) is satisfied. For $\nu<1$, we get

$$
\begin{aligned}
& \beta(k \epsilon)=-\frac{1}{\Delta_{-}}+\cdots, \\
& \widetilde{\beta}(k \epsilon)=-2^{2 \nu-1} \frac{\Gamma(\nu)}{\Gamma(1-\nu)}(k \epsilon)^{-2 \nu}+\cdots,
\end{aligned}
$$

whereas for $\nu>1$ we have

$$
\begin{aligned}
& \beta(k \epsilon)=-\frac{1}{\Delta_{-}}+\cdots, \\
& \widetilde{\beta}(k \epsilon)=-2(\nu-1)(k \epsilon)^{-2}+\cdots .
\end{aligned}
$$

As expected, $\beta$ and $\widetilde{\beta}$ have different asymptotic behaviors, and $\widetilde{\beta}$ diverges as we approach the boundary.

\section{CONCLUSIONS}

In this work, we have introduced a generalized AdS/CFT prescription, which is suggested by the results in $[6,7,10]$, and can consistently incorporate double-trace perturbations at the boundary CFT. This leads to a formalism in which we can perform explicit calculations. We have analyzed both minimally and nonminimally coupled cases, and obtained new results for both of them.

We have shown that the consistency of the formalism imposes a precise relation between the couplings $\beta$ and $\widetilde{\beta}$ [see Eq. (36)], and requires that at least one of them depends on the distance to the boundary. We have considered many possible couplings, and in all situations we have shown that to

\footnotetext{
${ }^{14}$ Recall here that $\Delta_{-}$depends on $\varrho$.
}

introduce a double-trace perturbation at the border is equivalent to adding a surface term to the usual action Eq. (13). This gives rise to different kinds of Dirichlet, Neumann, and mixed boundary conditions on the scalar field. In all cases, we have performed the explicit calculation of $\beta$ and $\widetilde{\beta}$ and of the corresponding surface terms. We have found that there exist particular values of $\beta$ and $\widetilde{\beta}$ which allow for irregular modes to propagate as well. We have computed the explicit expressions of such special couplings. We have shown that, as we get closer to the situation in which irregular modes are allowed to propagate, the coupling corresponding to the operator of conformal dimension $\Delta_{+}$diverges. This result is consistent with the statement in [6] that, as the coupling grows, the system approaches the condition that is suitable for quantization to get a field of dimension $\Delta_{-}$. In addition, we have shown that, when the constraints for which irregular modes propagate are satisfied, $\beta$ and $\widetilde{\beta}$ have different asymptotic behaviors, and the coupling corresponding to the operator of conformal dimension $\Delta_{+}$diverges as we approach the boundary.

We have also shown that there exist particular values of $\beta$ and $\widetilde{\beta}$ which require the introduction of new boundary conditions which have not been considered in [10]. We have included these new boundary conditions in our analysis.

In the particular nonminimally coupled case, we have also shown that the introduction of a double-trace perturbation at the boundary generates the natural extension of the GibbonsHawking surface term.

In general, we have shown that the introduction of double-trace perturbations can be understood in terms of the formulation in [10]. In particular, this enables us to relate the double-trace perturbations to the quantization which makes use of the canonical energy instead of the metrical one. Such quantization appears to be the natural one to be considered in the AdS/CFT context.

Throughout this article, we have only considered the simplest nontrivial case of multitrace perturbations, namely that of double-trace ones. It would be interesting to extend this formalism to the case of higher power trace perturbations. It should be noted that in such a case nonlinear boundary conditions would arise which would require us to consider a perturbative approach. More complex calculations would be involved to first extend the formulation in [10] and then to employ it to describe the more complicated multitrace perturbations. We also would like to be able to reproduce the constraints for which irregular modes propagate in the bulk and the couplings diverge by performing calculations exclusively on the boundary CFT side. It also would be interesting to understand the meaning of the generalized boundary conditions in the string theory context. This requires a more detailed study.

\section{ACKNOWLEDGMENTS}

I would like to thank J. Maldacena for helpful discussions. Special thanks to V. O. Rivelles for encouragement and a critical reading of the manuscript. This work was supported by FAPESP Grant No. 01/05770-1. 
[1] O. Aharony, M. Berkooz, and E. Silverstein, J. High Energy Phys. 08, 006 (2001).

[2] O. Aharony, M. Berkooz, and E. Silverstein, Phys. Rev. D 65, 106007 (2002).

[3] J.M. Maldacena, Adv. Theor. Math. Phys. 2, 231 (1998).

[4] O. Aharony, S.S. Gubser, J. Maldacena, H. Ooguri, and Y. Oz, Phys. Rep. 323, 183 (2000).

[5] E. D'Hoker, S.D. Mathur, A. Matusis, and L. Rastelli, Nucl. Phys. B589, 38 (2000).

[6] E. Witten, "Multi-Trace Operators, Boundary Conditions, and AdS/CFT Correspondence," hep-th/0112258.

[7] M. Berkooz, A. Sever, and A. Shomer, J. High Energy Phys. 05, 034 (2002).

[8] E. Witten, Adv. Theor. Math. Phys. 2, 253 (1998).

[9] S.S. Gubser, I.R. Klebanov, and A.M. Polyakov, Phys. Lett. B 428, 105 (1998).

[10] P. Minces and V.O. Rivelles, J. High Energy Phys. 12, 010
(2001).

[11] P. Breitenlohner and D.Z. Freedman, Ann. Phys. (N.Y.) 144, 249 (1982).

[12] P. Breitenlohner and D.Z. Freedman, Phys. Lett. 115B, 197 (1982).

[13] L. Mezincescu and P.K. Townsend, Ann. Phys. (N.Y.) 160, 406 (1985).

[14] I.R. Klebanov and E. Witten, Nucl. Phys. B556, 89 (1999).

[15] P. Minces and V.O. Rivelles, Nucl. Phys. B572, 651 (2000).

[16] G.W. Gibbons and S.W. Hawking, Phys. Rev. D 15, 2752 (1977).

[17] W. Mück, Phys. Lett. B 531, 301 (2002).

[18] D.Z. Freedman, S.D. Mathur, A. Matusis, and L. Rastelli, Nucl. Phys. B546, 96 (1999).

[19] W. Mück and K.S. Viswanathan, Phys. Rev. D 58, 041901 (1998). 